\title{
Motivated changes of auditory sensitivity in a simple detection task'
}

\author{
CHARLES S. WATSON ${ }^{2}$ AND BEN M. CLOPTON ${ }^{3}$ \\ DEFENSE RESEARCH LABORATORY, UNIVERSITY OF TEXAS
}

\begin{abstract}
Recently both neurophysiological and psychophysical theories have suggested that the sensitivity of receptors, or of the whole organism, may change through "efferent control" or as a result of "motivation." A psychophysical method has been devised to investigate changes in a listener's ability to detect signals in a noisy background, which are elicited "on demand" by the $E$, and when the time course of the changes may be in the order of seconds rather than minutes or hours. Observed effects, which are consistent with the hypotheses of active control of sensitivity, are found to be orderly but quite small, generally less than that associated with a $1-2 \mathrm{~dB}$ increase in the level of a tonal signal. While the average increment in performance is thus slight, it is found that the variance between $S s$ is significantly reduced when strong motivating stimuli are introduced. This reduction in variance may reflect an upper limit on the performance of real listeners, which might be fruitfully compared with the theoretical limits proposed in the theory of signal detectability.
\end{abstract}

Psychophysical results are always ambiguous inasmuch as we rely on the assumption that Os operate at or near their maximum capabilities in the research laboratory. That they do can only be a hope of the $E$, based on the fact that he has urged them to do as well as they can and, ad hoc, that they do about as well as any other Os under similar experimental conditions. We accept this implicit assumption, since psychophysical research produces good (but not perfect) agreement among the data from many Os, laboratories, and methods. Periodically, however, some new development requires that we question this concept. For example, two series of studies in recent years suggest moment-to-moment changes in the state of the $O$. A mathematical model of detection behavior, devised by Atkinson (1963) assumes that the $O$ changes his state as a function of both previous stimuli and previous responses. Also, several studies in neurophysiology have been concerned with a possible source of variation in the sensory state of the organism, the efferent fibers in the receptor pathways (Livingston, 1959; Hernandez-Peon, Scherrer, \& Jouvet, 1956). The latter developments are mentioned because of the recent tendency to invoke efferent "sensory tuning" to explain behavioral effects, even when there may be little systematic evidence that some of these effects exist. Particular reference is made to changes in sensitivity which are sometimes thought to be associated with states variously called arousal, activation, or alertness at one extreme, and habituation, depression, or boredom at the other. While performance in sensory tasks surely changes under these conditions, it is not at all clear that the source of such changes is a modification of sensitivity rather than a shift in the detection theorist's criterion of acceptance (Swets \& Sewall, 1963).

Swets and Sewall concluded from their own research, and from a thorough review of the literature, that there was no evidence for the fluctuations in state (sensitivity) proposed by Atkinson. Their work, however, like the studies they reviewed, was normal, molar psychophysical research. Atkinson's proposed fluctuations in sensitivity originated in a molecular psychophysical model, apparently intended to apply to trial-by-trial results. ${ }^{4}$ The possibility remains that shortduration changes in sensitivity may occur, changes in the order of seconds rather than minutes or hours or days, and that these fluctuations may be obscured in long-term averages like those presented by Swets and Sewall and most other modern psychophysical investigators.

Two types of transient fluctuations in sensitivity might exist. Periodic or aperiodic changes in the sensitivity of an $O$ may occur over time, independent of any activity on the part of the E. 5 Or, a second class of fluctuations might be those which could be described as "motivated," or potentially under the control of the $\mathrm{O}$ and, in turn, of the E. These latter would include changes in sensitivity induced through variations in the values and costs, or rewards and punishments, experienced by the $O$ during a sequence of psychophysical trials. The experiments reported here were designed to measure this second effect, in which enhanced performance might be elicited "on demand" by the $\mathrm{E}$.

The psychophysical method in these experiments involves special trials which are interpolated into a standard psychophysical sequence. Methods like it have been used by several investigators in studying the trial-by-trial effects of both "stimulus" (same-modality) and "nonstimulus" (other modality, or preexperimental) variables (Collier \& Verplanck, 1958; Greenberg \& Larkin, 1966). In such a procedure all trials other than the interpolated ones, and perhaps those immediately following interpolated trials, are regarded as the context, or frame. The purpose of these contextual trials is simply to maintain the $O$ in a standard state, from which deviations may be measured as a function of some variable introduced on the interpolated trials. We have referred to this general procedure as a psychophysical method of interpolated trials, as there appears to be no other descriptive term for it in the literature, although it has been used frequently by Helson (1964) in his studies of levels of adaptation. In all experiments reported below, the incentive to give a correct response on the interpolated trials was in some sense higher than on the contextual trials.

Asymptotic performance in typical detection experiments has been demonstrated within the first 10-20 min of listening. Gundy (1961), in an extensive study of training on detection performance, found that his listeners reached asymptotes within the first hour of listening, and that only a simple demonstration of the to-be-detected signal was required to reduce the time required for learning by one-half. In the present experiments we have attempted to elevate performance above the asymptotic levels established after a minimum of five days of practice ( $1 \mathrm{~h}$ per day).

\section{GENERAL METHOD}

\section{Listeners}

The listeners were 11 university students, each naive with respect to detection experiments and to the specific nature of the present research. All audiograms were $\pm 5 \mathrm{~dB}$ from audiometric zero (Davis \& Kranz, 1964), from 100 to $6000 \mathrm{~Hz}$. The listeners were paid $\$ 1.25$ per hour.

\section{Apparatus and Stimuli}

Auditory stimuli were generated and delivered to earphones by standard acoustic components. The signal to be detected in each experiment was a $500-\mathrm{Hz}$ sinusoid, $150 \mathrm{msec}$ in duration, which was presented at $66.5 \mathrm{~dB}$, relative to 0.0002 microbar. The signal was gated without respect for phase and with a rise-decay time of $25 \mathrm{msec}$. A background noise was 
continuously present in each experiment, with a uniform spectral level of $49.0 \mathrm{~dB}$ in the range from 100 to $3000 \mathrm{~Hz}$. The signals and the noise were presented in the same phase at the two earphones (NO-SO).

On each trial two observation lights flashed in sequence on the listener's console and the signal was presented, by a random schedule, during one of the lights. The observation lights were $175 \mathrm{msec}$ in duration, with $500 \mathrm{msec}$ between the termination of the first light and the onset of the second. The signal was temporally equidistant from the onset and termination of the light during which it was presented. A response interval of $1 \mathrm{sec}$ followed the second light, and the listener could press one of two response keys to indicate during which of the lights he believed the signal to have been presented. During this response time, an additional light flashed on when either of the keys was pressed, indicating that the response was firm enough to have been recorded, and that it was made in the allotted time. No information about the correctness of an individual response was given to the listener. The total time from the onset of one trial to that of the next was $3 \mathrm{sec}$ in all but Experiment 4, where it was $2.85 \mathrm{sec}$.

Responses were accumulated in banks of digital counters. Each bank of counters had four registers, one for each of the possible stimulus-response conjunctions. From 100 to 130 trials were included in each listening block. In the method of interpolated trials, additional incentive to make a correct response was introduced on a number of randomly selected trials in each block. A 4-in. square panel was illuminated in front of the listener $1 \mathrm{sec}$ prior to the first signal interval on each of these high-incentive trials (HITs), and this light was left on for the duration of the trial. Separate banks of counters were used to record the responses on single HITs, on each trial out of a sequence of HITs (Experiments 3 and 4), and on each of the three or four trials following a HIT or sequence of HITs, and an additional bank of counters recorded responses on other "normal" trials.

In Experiments 2-4 a shock could be applied to the listener, via electrodes attached to the ankle, when he did not respond correctly on HITs. The shock was maintained at approximately $1.6 \mathrm{~mA}$ from a constant current, $60-\mathrm{Hz}$ source, and always consisted of a single pulse, $200 \mathrm{msec}$ in duration.

\section{Procedure}

One experimental session was conducted per day. A session consisted of six blocks of trials, with approximately $5 \mathrm{~min}$ required for each block. A 2 -min rest was allowed after each block except the third, when the listeners rested for $5 \mathrm{~min}$.

\section{Training}

In each experiment the listeners were trained for a minimum of seven sessions prior to the introduction of HITs. While the number of days of training varied from experiment to experiment, the total number of training trials was always at least an order of magnitude greater than that $(400)$ found by Gundy to produce asymptotic performance in a similar detection task.

\section{EXPERIMENT 1}

\section{Method}

Complete details of the training procedures are described in each of these experiments. While such detail is often unnecessary, we believe that, in studies designed to demonstrate enhanced performance as a function of motivational variables, the readers will require more than the usual statement that "highly trained listeners were used in these experiments."

Sessions 1-8. Two listeners were trained in the detection task. Six blocks of 120 trials each were run per day, with demonstrations of the signal (noise reduced by $10 \mathrm{~dB}$ for five trials) preceding the first block on each day.
Table 1

The Percent of Correct Decisions in the Various Conditions of Experiment 1. $\dagger$

\begin{tabular}{llll} 
Condition & \multicolumn{2}{l}{$\begin{array}{l}\text { Subject } \\
\text { DT }\end{array}$} & Mean \\
\hline Training (last 5 days) & $72.7(3600)$ & $71.3(3600)$ & $72.0(7200)$ \\
Experimental sessions & & & \\
$\quad$ HITs & $72.2(320)$ & $71.5(320)$ & $71.85(640)$ \\
$\quad$ Normal trials & $72.9(1600)$ & $70.6(1600)$ & $71.75(3200)$ \\
\hline
\end{tabular}

t The numbers of trials on which the percentages are based are shown in parentheses. Listeners were offered financial rewards for enhanced performance on high-incentive trials (HITs); electrical shock was not used in this experiment.

Sessions 9-14. The listeners were told that the panel light would be illuminated occasionally just before a trial, and that the light should be interpreted as a request for maximum effort. They were further told that their performance on these trials, during which the panel light was illuminated, was the purpose of the whole experiment, and that these trials were the reason for all of the previous training. It was suggested that the research might have importance to the "defense efforts" and to "communication over noisy channels, as those used by men orbiting in space," and in general to the well-being of people in critical situations. Also, additional monetary reward was promised in the form of an additional hour's pay $(\$ 1.25)$ for each increase of $1 \%$ correct of their responses on HITs over the per cent correct during the last four training sessions. Twenty HITs were randomly interpolated in each block of 120 trials. A constraint on the random sequences required that at least three normal trials separate successive HITs.

\section{Results and Discussion}

The results of Experiment 1 are shown in Table 1, in the form of the per cent correct responses on normal trials and on HITs. No significant differences in performance are represented in these data. Also, the average performance on both normal trials and HITs was essentially the same as that on the last four days of training, supporting the conclusion that neither the motivating instructions, nor the promise of additional monetary reward, nor any extra effort that may have been made on HITs produced a measurable change in detection performance. This result, while demonstrating once again the adequacy of routine instructions to "do as well as you can," left open the possibility that still other forms of motivation could produce enhanced performance. The statistical significance of these and all other results in these experiments was estimated by assuming the variance of the proportion of correct responses to be $\mathrm{PQ} / \mathrm{N}$, and by using the normal approximation to the binomial (all Ns were greater than 100). While the assumption of trial-by-trial independence is almost certainly not met in these data, this test seems justified since failure to meet this assumption will result in a yet-more-conservative test.

In each of these experiments the dependent variable is an increment in the per cent of correct responses in the two-alternative, forced-choice psychophysical task (2AFC). From a psychometric function for this same task we can also describe an increment in performance in terms of the change in signal level which would typically give rise to it. There is no generally accepted form of this function, although there are a great number of determinations of it and theories about it. For the purposes of this paper we have referred our results to a form of the psychometric function described by Egan, Lindner, and McFadden (1965), $d^{\prime}=m\left(E / N_{0}\right)^{k}$. These authors found that, although differing between listeners, values of $k$ were not changed for a variety of binaural listening conditions. The psychometric functions shown in Fig. 1 are determined from this formula, and also by the unique transformation 


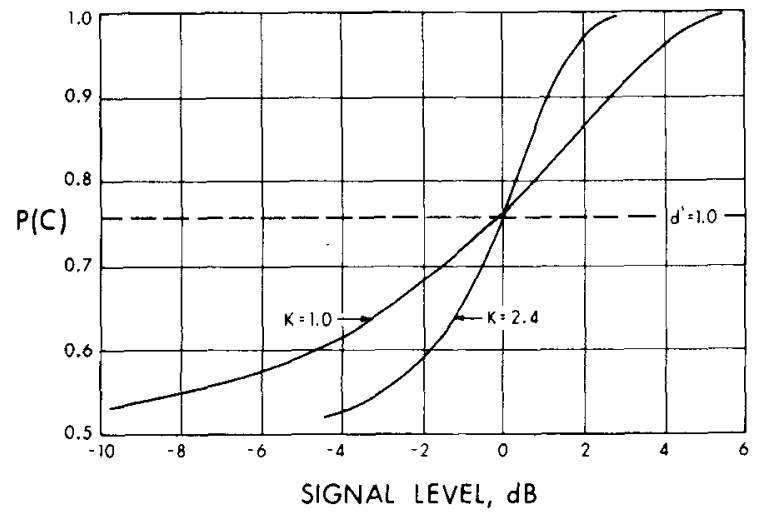

Fig. 1. Typical relations between the per cent of correct judgements, $\mathrm{p}(\mathrm{C})$, in two-alternative, forced-choice detection tasks and signal level in $\mathrm{dB}$, for the detection of a tonal signal in broadband noise. The more gradual function is typical of most detection research; the other is the steepest curve obtained in recent unpublished experiments by Watson and Lucas.

between $\mathrm{d}^{\prime}$ and the probability of a correct response in the two-alternative, forced-choice psychophysical procedure, as tabled by Elliott (1964).

Egan, Lindner, and $\mathrm{McF}$ adden reported values of $\mathrm{k}$ ranging between 1.0 and 1.5; however, Watson and Lucas (unpublished research) have recently found occasional values as high as 2.4 . Because of this lack of general agreement, we have shown psychometric functions for values of both 1.0 and 2.4. The approximate change of signal level which would yield a change in performance similar to that obtained through stronger motivation in the present experiments can be adequately estimated from this pair of functions. For example, the increment in signal level expected to yield a change from 70 to 80 per cent correct might be as great as 2.0 , or as little as $0.7 \mathrm{~dB}$ depending on the value of $\mathrm{k}$, but it would not likely fall outside this range.

\section{EXPERIMENT 2}

\section{Method}

Sessions 1-14. Three listeners were trained in the detection task, with blocks of 120 trials each, six blocks per session. They were instructed to "do as well as you can" and were given demonstrations of the signal, as in the first experiment.

Sessions 15-21. The listeners were told that "special" trials were to be introduced occasionally within each block, and that these trials would be signaled by the panel light. The general instructions about the importance of performance on these HITs was the same as in the previous experiment except that

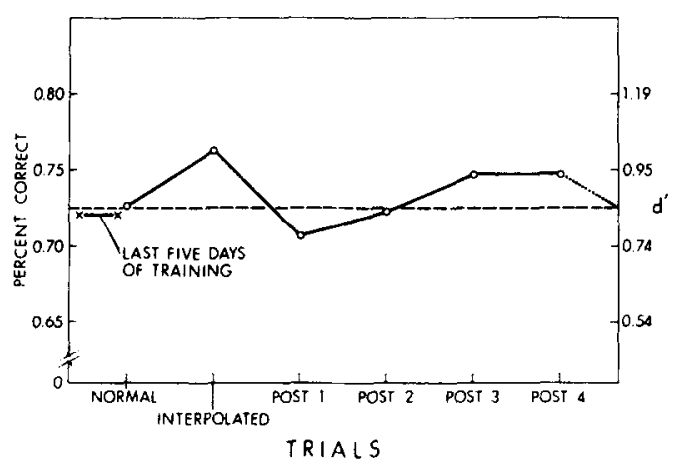

Fig. 2. Per cent correct judgements in Experiment 2, showing increment in average performance on interpolated high-incentive trials, reduced performance following these trials, and gradual return to better-than-average performance on the three trials following a high-incentive trial. Intertrial interval was $3 \mathrm{sec}$.

no additional monetary reward was promised. Instead, they were told (truthfully) that they would receive a shock to their ankles after each incorrect response on HITs. The shock was administered several times to each listener and, although they commented that it was quite unpleasant, they all agreed to continue in the experiment. (The listeners were told at this stage, in each experiment where shock was used, that they were welcome to resign without penalty if they had a strong aversion to even light electrical shock.) As in the first experiment 20 HITs were interpolated in each block of 120 trials for the remainder of the experiment.

\section{Results and Discussion}

The results of Experiment 2 are shown in Table 2 and in Fig. 2. Average performance on the HITs was significantly greater than (a) performance on "normal" trials, and (b) performance during the last five days of training $(p<.01)$. Reference to Fig. 1 shows that these small changes in performance are approximately what we would predict had we raised the signal level by $0.5-1.0 \mathrm{~dB}$. The performance on "normal" trials was not significantly different from that during the last five days of training. The difficulty in arbitrary definition of normal trials, because of the uncertain duration of the effects of the HITs, led us to calculate two other measures of performance: the per cent of correct responses on all trials during the experimental sessions, and the per cent correct on all trials other than the HITs. The first of these measures, as shown in Table 2, shows that overall performance

Table 2

The Percent of Correct Decisions in the Various Conditions of Experiment 2. +

\begin{tabular}{|c|c|c|c|c|}
\hline \multirow[b]{2}{*}{ Condition } & \multicolumn{3}{|c|}{ Subject } & \multirow[b]{2}{*}{ Mean } \\
\hline & $\mathrm{MH}$ & MA & MSJ & \\
\hline Training (last 5 days) & $72.8(2992)$ & $75.6(2999)$ & $70.9(2878)$ & $73.1(8869)$ \\
\hline \multicolumn{5}{|l|}{ Experimental Sessions } \\
\hline Normal trials & $73.5(2137)$ & $76.0(2134)$ & $68.5(2137)$ & $72.7(6408)$ \\
\hline High-incentive trial (HIT) & $78.0(432)^{*}$ & $79.1(431)$ & $71.3(432)$ & $76.1(1295)^{* *}$ \\
\hline Post-HIT-1 & $71.5(431)$ & $72.7(432)$ & $68.2(431)$ & $70.8(1294)$ \\
\hline Post-HIT-2 & $68.3(432)$ & $77.5(432)$ & $70.8(432)$ & $72.2(1296)$ \\
\hline Post-HIT-3 & $77.5(432)$ & $77.3(432)$ & $69.6(431)$ & $74.8(1295)$ \\
\hline Post-HIT-4 & $77.3(432)$ & $76.8(432)$ & $70.4(432)$ & $74.8(1296)$ \\
\hline Normal and Post-HIT trials & s $73.6(3864)$ & $76.0(3862)$ & $69.1(3863)$ & $72.9(11,589)$ \\
\hline $\begin{array}{l}\text { Overall, Experimental } \\
\text { Sessions }\end{array}$ & $74.0(4296)$ & $76.3(4293)$ & $69.2(4295)$ & $73.2(12,884)$ \\
\hline
\end{tabular}

*** Significantly greater than percent correct on normal trials:

$\left.{ }^{*}\right) p<0.05,0^{* *} p<0.01$

t The numbers of trials on which the percentages are based are shown in parentheses. 


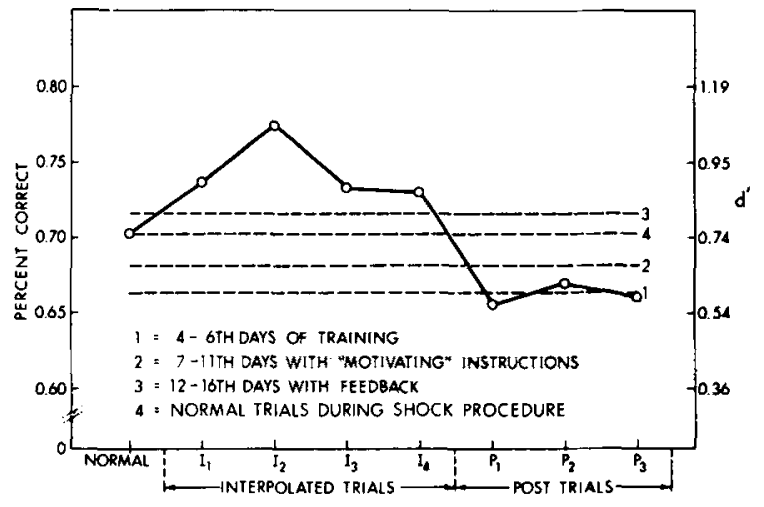

Fig. 3. Dashed lines show levels of performance during various stages of training, as indicated in the legend. Performance rose to a maximum on the second interpolated trial within sequences of four interpolated high-incentive trials, and then fell to lower than average performance following such sequences. Trial-by-trial recording was not carried out for enough trials to allow determination of the time at which performance returned to its normal level.

in the interpolated trials procedure was nearly identical to that in the last five days of training. The second supports a conclusion suggested by the data shown in Fig. 2, that the increment in performance on a single interpolated trial is followed by a reduction in performance.

There is some suggestion of a secondary period of enhanced performance at about the third trial $(9-10 \mathrm{sec})$ after a HIT, which might be maintained for about $6 \mathrm{sec}$. We were hesitant to conjecture further about the time course of enhanced performance since it is very likely related to the shocks which were delivered on $20-25 \%$ of the HITs. The shock may have caused the listeners to "attend" to their ankles rather than to the acoustic input on the trials immediately following those on which they were shocked. Even when shock was not delivered (after a correct response on HITs) there may have been some kind of conditioned startle response which might also serve to depress performance on subsequent trials. Whatever the source, it is clear that performance was depressed below both the overall average and the average on normal trials on the two trials immediately following a HIT. Whether enhanced performance could be maintained for longer than one trial ( $3 \mathrm{sec}$ ) could not be determined with this single-interpolated trial procedure, but the later period of improved performance suggested that this might be the case. For this reason, the third experiment was conducted.

\section{EXPERIMENT 3}

\section{Method}

Sessions 1-3. A new group of four listeners was trained in the detection task. The signal was demonstrated at a high signal-to-noise ratio for five trials at the beginning of each session. Six blocks of trials were run per day, with 120 trials per block.

Sessions 4-6. No further demonstrations of the signal were given, and the listeners were urged to "do as well as you can."

Sessions 7-11. Additional instructions were given to the listeners, repeating the items used in the first experiment concerning the "defense effort," and critical listening situations. In general they were given the strongest verbal motivation that the Es could contrive.

Sessions 12-16. After each block of trials the listeners were told how well they had done on that block, relative to their average performance in Sessions 10-12. The feedback after each block consisted of the statements, "that was all right," for performance $\pm 4 \%$ from their previous average, "very good!" for performance $5 \%$ or more above average and, "you didn't do very well that time," for lower performance.
Sessions 17-24. Sequences consisting of four successive interpolated trials were introduced into each block, using the same shock incentive as in the second experiment. Six sequences of four HITs per sequence were interpolated in each block, in addition to 100 normal trials.

Sessions 25-26. The final two days of the experiment were devoted to a control condition in which no HITs were presented and no special instructions were given other than the usual "do as well as you can."

\section{Results and Discussion}

The effects of the different types of instructional motivation and feedback (Sessions 4-16) are shown by the horizontal lines of Fig. 3, and in Table 3. The largest of these effects was that obtained by telling the listeners how well they had done, immediately after each block of trials (Sessions 12-16). The resulting average increase in per cent correct, 3.7 , over that with motivating instructions, is significant at well beyond the 0.01 level of confidence. This improvement in performance as a function of block-by-block feedback bears special discussion, as "knowledge of results" has not generally had this effect in detection research. In particular, Gundy (1961) and Carterette and Wymann (1962) have reported that in detection tasks similar to the one described here, trial-by-trial feedback has either no effect or a negative one on asy mptotic performance. Schoeffler (1965) developed a model for learning over sequences of psychophysical trials which interprets a decrement in detection resulting from trial-by-trial feedback as an effect of induced variability in the response criterion. That is, the listener occasionally samples a signat-like instance of noise alone, and responds "signal," or a noise-like instance of signal plus noise and responds "noise." In these cases the feedback (information as to what was actually presented on these trials) will disagree with his response, even though his response might have been based in an optimal detection strategy. This disagreement might lead the listener to modify his strategy, or to adjust his response criterion. If he adjusts his criterion each time response and feedback disagree, the effect is equivalent to adding more variance to the noise, and thus reduces the overall probability of correct responses. However, human listeners can use block-by-block feedback to good effect, as shown by the improvement when this was introduced (Sessions 12-16) and also by the fact that performance returned to the prefeedback level when feedback was deleted (Sessions 25 and 26, and on "normal" trials in Sessions 17-24). These experiments were not specifically designed to study the effects of feedback, and the effects related to this variable remain equivocal.

During the interpolated-trials sessions, performance reached a maximum on the second HIT in the sequences of four HITs for three out of the four listeners, and on the first trial for the other. The average performance on the second interpolated trial was significantly greater than that during normal trials $(p<.001)$, and this enhancement was about what would be expected from a 1.5-2.0 dB increase in signal level (see Fig. 1). The listener (DP) who showed a maximum on the first trial in the HIT sequences had higher overall performance than the other listeners, and in the Es' judgement she was the most sensitive member of this group with regard to the shock. Depressed performance was again observed after the interpolated trials, lasting at least through the three post-HIT trials on which separate responses were recorded.

This experiment supported the hypothesis that the period of enhanced performance would be longer in duration if several HITs were presented in sequence, rather than singly, and that the maximum performance with sequences of HITs would be greater than with single interpolated trials. The increments in performance were still small, however, and Experiment 4 was conducted to investigate one factor which might have tended to reduce performance under the design of 
Table 3

The Percent of Correct Decisions in the Training and Experimental Conditions of Experiment 3. $\dagger$

\begin{tabular}{llllll}
\hline & & & \multicolumn{3}{c}{ Subject } \\
Condition & PF & JPr & DP & JPu & Mean \\
\hline Preliminary training & $62.8(1606)$ & $62.6(1727)$ & $69.1(1852)$ & $70.6(1734)$ & $66.3(6919)$ \\
$\begin{array}{l}\text { Training with motivating } \\
\text { instructions }\end{array}$ & $64.0(2963)$ & $63.3(3086)$ & $74.0(3090)$ & $70.9(2843)$ & $68.1(11982)$ \\
Training with block-by- & $70.8(3086)$ & $68.1(2954)$ & $75.7(3094)$ & $72.7(2920)$ & $71.8(12054)$ \\
$\quad$ block feedback & & & & & \\
Experimental Sessions & & & & & \\
Normal trials & $70.8(2863)$ & $63.9(2612)$ & $75.8(2867)$ & $70.8(2739)$ & $70.3(11081)$ \\
HIT-1 & $75.6(208)$ & $66.7(197)$ & $78.9(209)$ & $74.1(204)$ & $73.8(818)^{*}$ \\
HIT-2 & $78.3(206)^{*}$ & $76.9(192)^{* * *}$ & $77.8(210)$ & $76.8(201)$ & $77.5(809)^{* * *}$ \\
HIT-3 & $72.9(209)$ & $75.2(198)^{* * *}$ & $74.4(210)$ & $70.8(200)$ & $73.3(817)$ \\
HIT-4 & $72.8(203)$ & $70.0(198)$ & $75.0(209)$ & $74.1(199)$ & $73.0(809)$ \\
$\quad$ Post HIT-1 & $73.3(207)$ & $50.3(190)$ & $73.8(210)$ & $65.3(204)$ & $65.7(811)$ \\
Post HIT-2 & $67.8(209)$ & $57.3(197)$ & $74.4(210)$ & $68.3(203)$ & $67.0(819)$ \\
$\quad$ Post HIT-3 & $67.7(210)$ & $58.0(197)$ & $69.7(210)$ & $68.8(204)$ & $66.1(821)$ \\
All HITs & $74.9(826)$ & $72.2(785)$ & $76.5(838)$ & $74.0(804)$ & $74.4(3253)$ \\
$\quad$ Normal and Post-HIT & $70.6(3489)$ & $62.4(3196)$ & $7 i .2(3497)$ & $70.0(3358)$ & $69.6(13532)$ \\
$\quad$ trials & & & & & \\
Overall, Experimental & $71.4(4315)$ & $64.2(3981)$ & $75.5(4335)$ & $70.9(4154)$ & $70.5(16785)$ \\
$\quad$ sessions & & & & & \\
Post-experimental sessions & $66.5(1236)$ & $65.8(1239)$ & $75.6(1240)$ & $71.4(1235)$ & $69.8(4950)$ \\
\hline
\end{tabular}

*, *** Significantly greater than percent correct on normal trials: $\left({ }^{*}\right) p<0.05,\left(^{* * *}\right) p<0.001$

+ The numbers of trials on which the percentages are based are shown in parentheses. High-incentivetrials (HITs) were presented in sets of four in this experiment.

Experiment 3. In Experiment 3 the shock was delivered to the listener after some of the HITs, and therefore, on the trials preceding some of the HITs. As suggested earlier, the immediate effect of the shock might be to attract attention to the ankle rather than to the auditory input. The last experiment was designed to eliminate the possibility of shock on any trial immediately preceding a HIT.

\section{EXPERIMENT 4}

\section{Method}

Sessions 1-8. Two naive listeners were trained under the normal instructions, with blocks of 130 trials, and with six such blocks per daily session. The time from the onset of one trial to the onset of the next was shortened to $2.85 \mathrm{sec}$ in this experiment, in an attempt to reduce listener fatigue during the blocks of trials.

Sessions 9-13. The listeners were given an evaluation of their performance after each block of trials, following the same procedure used in Sessions 13-16 of Experiment 3.

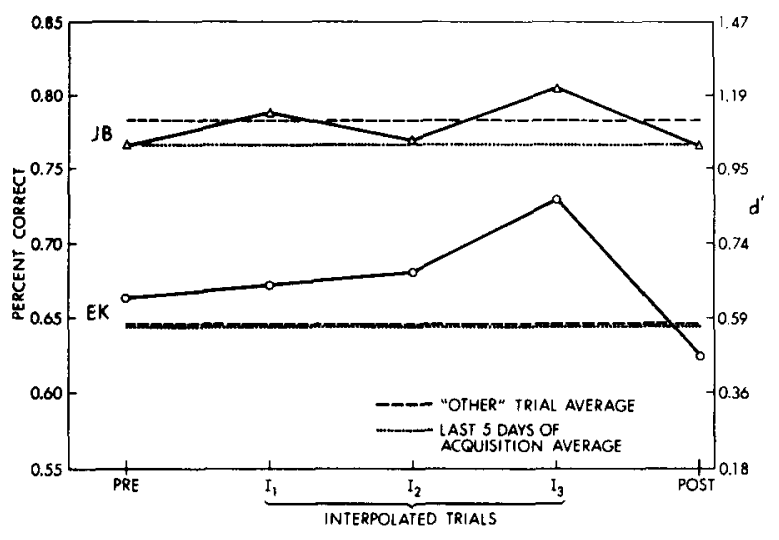

Fig. 4. Performance for the two listeners of Experiment 4 showing that a maximum is reached on the third interpolated trial (the last), when no interpolated trial was ever preceded by a trial on which shock was delivered.
Sessions 14-19. Sequences of one, two, or three HITs were randomly interpolated in each block of trials. Five sequences of each length were included in each block of trials, that is, 30 HITs and 100 normal trials per block. Shock was only introduced following an error on the last of a series of HITs, or on the third out of a sequence of three, the second out of two, or after an error on a single HIT. By this procedure it was not possible for an interpolated trial to follow a trial on which shock was delivered, although the threat of shock was present on every trial of such a sequence.

\section{Results and Discussion}

The results for the two listeners of Experiment 4 are displayed in Fig. 4 and in Table 4. Compared to the listeners in the earlier experiments both $\mathrm{JB}$ and EK showed even smaller increments on HITs [significant improvement above that in Sessions $9-13$ for EK $(p<.05)$ but not for JB]. The largest increment for each of these listeners, however, occurred on the third interpolated trial out of the sequence of three. This result gives some support to the hypothesis that in Experiment 3 shock on earlier trials had had a depressing effect on performance following shock. It is possible that the overall effectiveness of the shock was reduced due to the fewer number of shocks experienced by the listeners in this experiment. No strong conclusion can be reached with respect to the temporal course of enhanced performance on interpolated trials, except that the enhancement may increase at least for as long as the 9 sec between the appearance of the panel light and the third interpolated trial.

\section{SUMMARY AND CONCLUSIONS}

These experiments were designed to measure increments in auditory sensitivity produced by strong incentive to be correct, as indicated by variations in performance. Highincentive trials were interpolated in sequences of normal trials, in order that even short-duration changes in performance might be observed. Each of the nine listeners tested under this procedure showed an increment in performance on the high-incentive trials, when compared to asymptotic performance which had been maintained during the last several days 
Table 4

The Percent of Correct Decisions in the Training and Experimental Conditions of Experiment 4. $\uparrow$

\begin{tabular}{|c|c|c|c|}
\hline Condition & JB & $\begin{array}{r}\text { Subject } \\
\text { EK }\end{array}$ & Mean \\
\hline Training (last 5 days) & $76.7(3192)$ & $64.7(3120)$ & $70.7(6362)$ \\
\hline \multicolumn{4}{|l|}{ Experimental Sessions } \\
\hline Normal & $78.2(2520)$ & $64.7(2448)$ & $71.6(4968)$ \\
\hline Pre-HIT trial & $76.5(541)$ & $66.3(534)$ & $71.4(1075)$ \\
\hline HIT-1 & $79.0(537)$ & $67.2(518)$ & $73.2(1055)$ \\
\hline HIT-2 & $76.9(360)$ & $68.0(344)$ & $72.6(704)$ \\
\hline HIT-3 & $80.6(180)$ & $73.0(174)^{*}$ & $76.8(354)$ \\
\hline Post-HIT trial & $76.7(533)$ & $62.3(520)$ & $69.6(1053)$ \\
\hline All HITs & $77.9(4671)$ & $65.5(4538)$ & $71.8(9209)$ \\
\hline All non-HITs & $78.6(1077)$ & $68.4(1036)$ & $73.6(2133)$ \\
\hline $\begin{array}{l}\text { Overall, experimental } \\
\text { sessions }\end{array}$ & $77.7(3594)$ & $64.6(3502)$ & $71.3(7096)$ \\
\hline
\end{tabular}

* Significantly greater than percent correct on normal trials, $p<0.05$ $\dagger$ The numbers of trials on which the percentages are based are shown in parentheses. High-incentive-trials (HITs) were presented in sets of one, two, or three, and the possibility of shock following an incorrect response only existed for the final HIT in a set.

of a week or more of training on this same task. The average increment, however, was only the equivalent of about a 0.5-1.0 dB increase in signal level, and the distribution of these increments was positively skewed.

Perhaps the simplest interpretation of the individual differences in increments in performance is that some people work harder than others under normal instructions. Listeners may work at levels of efficiency which, although appearing to be asymptotic performance, represent varying fractions of their potential peak efficiency. This interpretation implies that the size of the increment in performance on high-incentive trials must be a function of performance on normal trials. Fig. 5 shows the relation between the maximum increment on HITs and the performance on normal trials, for the nine listeners tested under the shock conditions.

Variability of performance for the several listeners in these experiments was considerably smaller on high-incentive than on normal trials. The associated standard deviations, in per cent, were 2.94 and 5.00 , which are significantly different $(p<0.01)$. The relation between normal performance and the increment in performance on high-incentive trials is described by a regression line in Fig. 5 . The Spearman coefficient of correlation between these two variables is -0.84 . This negative correlation and the associated reduction in variance with increased motivation suggest that the listeners may be working against an upper limit on performance, a limit which they could not exceed with any further motivation. This limit can be estimated by the intersection of the regression line of Fig. 5 with the abscissa, indicating that a listener who achieves $81 \%$ correct has "given his all." Comparing this level of performance to data for a large number of highly trained listeners in our laboratory, we found that it is close to the best performance obtained for even our most experienced people. In fact, none of our previous listeners had produced average performance as high as this. Application of this estimate of the performance of an "ideal" human listener to the estimation of some other auditory parameters might prove a useful adjunct to other current theoretical approaches to detection.

In summary, these small, motivation-induced increments in per cent of correct judgements show that listeners are capable of momentary enhancement of detection performance, lasting at least $9 \mathrm{sec}$, but also that the average increments are not large enough to be an important consideration in most (auditory) psychophysical research.

No support was found for theories which attribute graded psychophysical performance to wide fluctuations in sensitiv- ity; if such fluctuations do exist, they are not under the control of the listener. As to whether or not the sensitivity of the auditory system may be modified through auditory efferents, or by any other means, we conclude that the simple request that the listener "do as well as he can" leads to near-peak performance, for most listeners. As severe an additional motivator as an electric shock delivered immediately after an error results in only slightly better performance than does this instruction.

These results do not mean, of course, that psychophysical performance cannot be modified by motivational variables, even drastically modified. The probabilities of various responses can be altered easily through changes in the relative values and costs associated with the responses, as shown in many experiments in signal detection theory (Green \& Swets, 1966). The experiments described here have shown only that while criterion (response strategy) may be strongly affected by motivation, measures which are not confounded with criterion are only very slightly subject to such variation. This conclusion refers to the range of motivation between the instruction to "do well," and stronger incentives, as the promise of considerable additional pay, or punishment by electric shock. Whether changes in sensitivity may occur with lower levels of motivation remains as a separate experimental question.

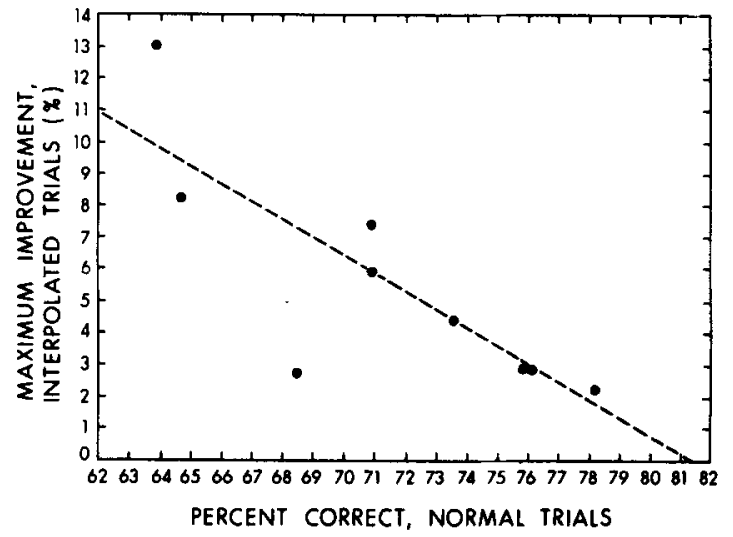

Fig. 5. Relation between per cent correct on normal trials and maximum increment in per cent correct on high-incentive trials, for listeners in Experiments 2-4. Correlation between normal performance and increment in performance is -0.84 , suggesting that listeners may be approaching an upper limit which they could not exceed with further motivation.

\section{REFERENCES}

ATKINSON, R. C. A variable sensitivity theory of signal detection. Psy chological Review, 1963, 70, 91-106.

CARTERETTE, E. C., \& WYMAN, M. J. Application of a Markov Learning Model to a simple detection situation involving social pressure. In J. Criswell, H. Solomon, and P. Suppes (Eds.), Mathematical methods in small group processes. Stanford: Stanford University Press, 1962. Pp. 74-100.

COLLIER, G., \& VERPLANCK, W. S. Nonindependence of successive responses at the visual threshold as a function of interpolated stimuli. Journal of Experimental Psychology, 1958, 55, 429-437.

DAVIS, H., \& KRANZ, F. W. Reference zero for pure-tone international standard audiometers. Journal of Speech \& Hearing Research, 1964, 7,7-16.

EGAN, J. P., LINDNER, W. A., \& McFADDEN, D. Masking-level differences and the form of the psychometric function. Joumal of the Acoustical Society of America, 1965, 37, 1181.

EKMAN, G., \& FRANZÉN, O. Measurement of the underlying process in auditory fluctuations. Studia Psy chologica, VIII, 1966, 2, 96-105.

EKMAN, G., \& LINDMAN, R. Measurement of the underlying process in perceptual fluctuations. Vision Research, 1962, 2, 253-260.

ELLIOTT, P. B. Tables of d'. Technical Report No. AFCRC-TR-59-55, 1959, University of Michigan, Electronics Defense Group.

GODFREY, R. R. Stability of signal detection ability. Unpublished M.A. thesis, University of Texas, 1966. 
GREEN, D. M. Consistency of auditory detection judgements. Psychological Review, 1964, 71, 392-407.

GREEN, D. M., \& SWETS, J. A. Signal detection theory and psychophysics. Wiley, 1966.

GREENBERG, G. Z., \& LARKIN, W. D. Frequency-selective detection of signals in noise. Journal of the Acoustical Society of America, $1966,39,1247$.

GUNDY, R. F. Auditory detection of an unspecified signal. Journal of the Acoustical Society of America, 1961, 33, 1008-1012.

HELSON, H. Adaptation-level theory, an experimental and systematic approach to psychology. New York: Harper and Row, 1964.

HERNANDEZ-PEON, R, SCHERRER, H., \& JOUVET, M. Modification of electric activity in cochlear nucleus during "attention" in unanesthetized cats. Science, 1956, 123, 331-332.

LIVINGSTON, R. B. Central control of receptors and sensory transmission systems. In H. W. Magoun (Ed.), Handbook of Physiology. Sect. 1, Vol. I Washington, D.C.: American Physiological Society, 1959. Chap. XXXL. Pp. 741-760.

SCHOEFFLER, M. S. Theory for psychophysical learning. Journal of the Acoustical Society of America, 1965, 37, 1124-1133.

SWETS, J. A., \& SEWALL, S. T. Invariance of signal detectability over stages of practice and levels of motivation. Joumal of Experimental Psychology, 1963, 66, 120-126.

\section{NOTES}

1. The research reported here was conducted at the Defense Research Laboratory, The University of Texas, through the support of contracts with the U.S. Navy, Bureau of Ships, and with the National Aeronautics and Space Administration. Support for preparation of the manuscript was provided by the National Institutes of Health, through the Central Institute for the Deaf. The authors are indebted to Prof. Lloyd A. Jeffress for his many valuable comments.

2. Address: Central Institute for the Deaf, St. Louis, Missouri 63110.
3. Address: Departinent of Psychology and Department of Physiology and Biophysics, University of Washington, Seattle, Washington 98105. The experimental work described in this article was carried out by the second author in partial fulfillment of the requirements of the Master's degree in psychology from the University of Texas, Austin.

4. Green (1964) has distinguished between molar and molecular psychophysics on the basis of models and related research that deal, on the one hand, with averages from long series of psychophysical data, and on the other with trial-by-trial effects. The preponderance of psychophysical research has ignored molecular issues, probably under the assumption that such effects account for little of the total variance and are peripheral to the study of sensory capability. Since the relative contributions of variables other than the immediate stimulus (previous stimuli, previous responses, motivation, feedback, and so on) are inversely related to the strength of that stimulus, such an attitude was defensible in classical experiments. These experiments generally dealt with stimuli at or above statistically defined threshold levels, where such "nonstimulus" effects are probably minimal.

5 . We have not observed such periodicities in detection performance in our laboratory. In particular, in an analysis by Godfrey (1966), no regular periodicities were found in a rating experiment, and in fact the only sequential effects were a very slight tendency for responses to be repeated, which lasted over about three trials $(12 \mathrm{sec})$. However, we are aware of conflicting results by Ekman and Lindman (1962) and by Ekma.1 and Franzén (1966), who report periodic fluctuations of the threshold in both auditory and visual experiments. It is difficult to determine whether the fluctuations of threshold reported in experiments like those of Ekman et al are genuine changes in sensitivity, or are related to some other variable also affecting the O's decision.

(Accepted for publication November 5, 1968.) 